\title{
Conductance fluctuations at the integer quantum Hall plateau transition
}

\author{
Sora Cho \\ Department of Physics, University of California, Santa Barbara, California 93106 \\ Matthew P.A. Fisher \\ Institute for Theoretical Physics, University of California, Santa Barbara, California 93106 \\ and Department of Physics, University of California, Santa Barbara, California 93106
}

(September 17, 2018)

\begin{abstract}
We study numerically conductance fluctuations near the integer quantum Hall effect plateau transition. The system is presumed to be in a mesoscopic regime, with phase coherence length comparable to the system size. We focus on a two-terminal conductance $G$ for square samples, considering both periodic and open boundary conditions transverse to the current. At the plateau transition, $G$ is broadly distributed, with a distribution function close to uniform on the interval between zero and one in units of $e^{2} / h$. Our results are consistent with a recent experiment by Cobden and Kogan on a mesoscopic quantum Hall effect sample.
\end{abstract}

PACS: 05.30.-d, 73.23.-b, 73.40.Hm, 74.20.-z

\section{INTRODUCTION}

One of the early surprises of the burgeoning field of mesoscopic physics some ten years ago was the observation of large conductance fluctuations in small metallic samples. ing their size were found to exhibit sample (or field) specific fluctuations in their conductance. The magnitude of the fluctuations - of order $e^{2} / h$ - was essentially independent of the mean conductance, leading to the name "universal conductance fluctuations." Theoretical explanations are based on models of diffusing electrons, in which localization effects can be ignored $\mathbf{3}$ Generally, this requires that the mean conductance is much larger than $e^{2} / h$, a condition fulfilled in the experiments.

One of the striking featufes of the plateau transitions in the quantum Hall effect, 1 国 is that the magnitude of the macroscopic longitudinal conductivity $\sigma_{x x}$ is both metallic - independent of temperature as $T \rightarrow 0-$ and of order $e^{2} / h$. Conventional localization effects are inoperative due to the strong applied magnetic field. For the transition from insulator to the first filled Landau level, the experimental valued $\$$ for the conductivity tensor are consistent with

$$
\sigma_{x x}=\sigma_{x y}=\frac{1}{2} \frac{e^{2}}{h} .
$$

These macroscopic conductivities are self-averaged, since the sample sizes are much bigger than the phase coherence length. Several authors have given theoretical arguments in support of these values, 10 although it is unclear that the averaging process is appropriate to the experiment.

Recently Cobden and Kogan have measured the conductance of a small_quantum Hall effect sample, in the mesoscopic regime.10 They find large fluctuations in a two-terminal conductance near the plateau transitions, as they vary the carrier density with a gate voltage.
Specifically, the conductance seems to be almost uniformly distributed on the interval between zero and one in units of $e^{2} / h$. In striking contrast to conventional metallic samples, the magnitude of the conductance fluctuations is comparable to the mean conductance, $\bar{G} \approx$ $(1 / 2) e^{2} / h$.

In this paper we compute the conductance fluctuations employing a simple network model of the inter-ger quantum Hall effect (IQHE) plateau transition.11.12 We extract a mesoscopic two-terminal conductance and its sample (and field) specific fluctuations. Our results are entirely consistent with the Cobden and Kogan experiment. Right at the transition, the conductance distribution function is essentially uniform on the interval from zero to one in units of $e^{2} / h$.

Our paper is organized as follows. In Sec. II we briefly review the network model, specifying the appropriate geometry and boundary conditions. The results for the conductance and its distribution are presented in Sec. III. Sec. IV is devoted to a brief discussion.

\section{THE NETWORK MODEL}

To model the IQHE plateau transition, employ Chalker and Coddington's network model.13 In this model the interactions between the electrons are ignored. In their original formulation, the impurity potential was assumed to be slowly varying on the scale of the magnetic length. The semiclassical trajectories moving along equipotentials were modeled via ballistic chiral propagation along the links of a network. Quantum tunneling at saddle points between nearby equipotentials was incorporated via tunneling at node parameters, connecting two incoming and two outgoing links. For simplicity, the nodes and links were placed on a regular (square) lattice. Randomness was incorporated via phase factors for 
propagation along the links, which were assumed to be independent and uniformly distributed between 0 and $2 \pi$.

In this paper we focus on the behavior near the plateau transition. Being a continuous (second order) phase transition, we expect that universal critical properties (including conductance fluctuations) should not depend on details of the model. Thus, for example, the results obtained should also apply to systems for which the potential is not varying slowly on the scale of the magnetic length. Extensive numerical simulations that have extracted the critical exponent $\nu$ for the diverging localization length support this supposition. For example, Lee, Wang, and Kivelson 14 have shown that inclusion of random scattering at the nodes gives the same for $\nu$ as in Chalker's original random-phase model.13 Moreover, consistent estimates for $\nu$ have also been obtained from other numerical approaches, such as Thouless number studies of lowest Landau level Hamiltonians.12 12 A more serious concern is the legitimacy of ignoring Coulomb interactions between the electrons. It is conceivable that interactions - particularly long-ranged Coulomb forces might be a "relevant" perturbation at the noninteracting phase trangition (fixed point), leading to new critical properties 15,16 However, experimental measurements of $\nu$ seem to coincide with the non-interacting value. Being practical, we ignore Coulomb interactions, and adopt Chalker and Coddington's network model.13

To be specific, we study a square lattice network of nodes and links, as depicted in Fig. 1. The network is connected to two leads - to the right and left. The distance between the leads, measured in units of the network lattice spacing, is denoted by $L_{x}$. Of interest is the twoterminal conductance between these two leads. The sample has a width, denoted $L_{y}$, in the transverse direction. We consider two boundary conditions in the transverse direction: (i) periodic boundary conditions and (ii) open boundary conditions. The case of open boundary conditions corresponds clpsely to the experimental geometry of Cobden and Kogan.10 In this case, edge states dominate the transport in the IQHE plateau.

Quantum tunneling at each node is represented by a 2 by 2 matrix,

$$
\left(\begin{array}{c}
w_{\text {out }} \\
w_{\text {in }}
\end{array}\right)=\left(\begin{array}{cc}
\cosh \theta & \sinh \theta \\
\sinh \theta & \cosh \theta
\end{array}\right)\left(\begin{array}{c}
v_{\text {in }} \\
v_{\text {out }}
\end{array}\right),
$$

where $v$ and $w$ represent complex amplitudes for incoming and outgoing electron waves to the right and left, respectively, of a given node (see Fig. 1). By construction, this matrix conserves the current, $\left|w_{\text {in }}\right|^{2}+\left|v_{\text {in }}\right|^{2}=$ $\left|w_{\text {out }}\right|^{2}+\left|v_{\text {out }}\right|^{2}$. The node parameter $\theta$ determines the degree of backscattering at the node. So, for example, an incident wave, say $w_{\text {in }}$, is back-scattered into $w_{\text {out }}$ with probability $\tanh ^{2}(\theta)$. To make the model invariant under a $\pi / 2$ spatial rotation, the node parameters take two values, $\theta_{1}$ and $\theta_{2}$, in alternating columns, and are chosen to satisfy the condition $\sinh \left(\theta_{1}\right) \sinh \left(\theta_{2}\right)=1$. Randomness is incorporated via random phase factors along links.
From symmetry, the plateau transition occurs when $\tanh \left(\theta_{1}\right)=\tanh \left(\theta_{2}\right)$, or $\theta=\theta_{c}=\ln (1+\sqrt{2})$. It is thus convenient to define a variable $\Delta$, which measures the "distance" to the transition

$$
\Delta=\tanh \left(\theta_{1}\right)-\tanh \left(\theta_{2}\right) .
$$

This parameter lies in the range $-1 \leq \Delta \leq 1$, and vanishes right at the plateau transition.

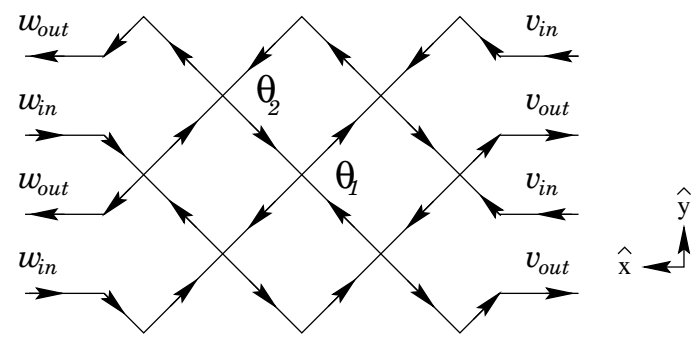

FIG. 1. Schematic representation of the network model for a square sample with size $L_{x}=L_{y}=4$. The arrows indicate the direction of wave propagation along the links, and $\theta_{1}$ and $\theta_{2}$ specify scattering at the nodes. The two-terminal conductance $G$ is measured between the right and left leads.

For open boundary conditions in the transverse $(y)$ direction, the nodes on the top and bottom edges are modified to be

$$
\left(\begin{array}{c}
w_{\text {out }} \\
w_{\text {in }}
\end{array}\right)=\left(\begin{array}{ll}
1 & 0 \\
0 & 1
\end{array}\right)\left(\begin{array}{c}
v_{\text {in }} \\
v_{\text {out }}
\end{array}\right)
$$

so that $w_{\text {out }}=v_{\text {in }}$ and $w_{\text {in }}=v_{\text {out }}$. In this case, the boundary breaks (lowest Landau level) particle-hole symmetry, $\Delta \rightarrow-\Delta$, just as the edges do in a real physical system. In the Hall plateau phase, $\Delta>0$, extended edge states confined to the top and bottom boundaries of the sample are expected. In the localized insulator, corresponding to $\Delta<0$, all states are localized, even near a boundary. In real systems, the presence of an edge state accounts naturally for the quantized Hall conductivity.

With transverse periodic boundary conditions, all nodes (in each column) are identical. In this case, there are of course no edge states. The two-terminal conductance is only sensitive to extended bulk states, present at the plateau transition. The finite system is particlehole symmetric $(\Delta \rightarrow-\Delta)$, as will be clear from the numerical results.

We compute numerically the total transfer matrix $T$ in the x direction, for a network of width $L_{y}$ and length $L_{x}$. This $L_{y}$ by $L_{y}$ matrix relates the incoming and outgoing amplitudes in one lead - the $v$ 's - to the amplitudes in the other lead - the w's, and can be written schematically as $W=T V$, where $W$ and $V$ are vectors with $L_{y}$ elements. To extract the two-terminal conductance $G$, it is useful to write $T$ in the form

$$
\left(\begin{array}{c}
W_{\text {out }} \\
W_{\text {in }}
\end{array}\right)=\left(\begin{array}{cc}
A & B \\
C & D
\end{array}\right)\left(\begin{array}{c}
V_{\text {in }} \\
V_{\text {out }}
\end{array}\right),
$$


where $V_{\text {in }}$ denotes an $L_{y} / 2$ column vector of the incoming amplitudes in the right lead, $V_{\text {out }}$ the outgoing, and similarly for the other lead. This can be inverted to obtain the $S$ matrix relating all the incoming to outgoing modes, as

$$
\left(\begin{array}{c}
W_{\text {out }} \\
V_{\text {out }}
\end{array}\right)=\left(\begin{array}{ll}
B D^{-1} & A-B D^{-1} C \\
D^{-1} & -D^{-1} C
\end{array}\right)\left(\begin{array}{c}
W_{\text {in }} \\
V_{\text {in }}
\end{array}\right) .
$$

The two-terminal conductance can be expressed in terms of the $L_{y} / 2$ by $L_{y} / 2$ transmission matrix $t$, which relates the incoming amplitudes of one lead to the outgoing amplitudes in the other lead: $V_{\text {out }}=t W_{\text {in }}$. Thus we have $t=D^{-1}$. The two-terminal conductance follows readily from $t$ as. 17

$$
G=\frac{e^{2}}{h} \operatorname{tr}\left[t t^{+}\right]
$$

Below we focus on the two-terminal conductance for square samples of size $L=L_{x}=L_{y}=4,8,16$, and 24 . The distribution function is obtained by evaluating $G$ for many different samples. We typically take $5 \times 10^{4}$ samples.

\section{RESULTS}

The mean conductance, denoted $\bar{G}$, is obtained by averaging $G$ over a large number of samples. In Fig. 2(a), $\bar{G}$ is plotted versus the control parameter $\Delta$, for four different sample sizes, all with periodic boundary conditions in the transverse direction. (In this and later figures, $G$ is plotted in units $e^{2} / h$.) As expected the mean conductance is largest at the plateau transition, $\Delta=0$, reflecting transport via bulk delocalized states, and falls off towards zero away from the transition. For the larger system sizes, the peak in $\bar{G}$ narrows. With open boundary conditions, the mean conductance rises from zero to one, as shown in Fig. 2(b). As the sample size increases, the "step" becomes less and less rounded. Together, the two sets of data for $\bar{G}$, resemble experimental plots of the macroscopic conductivities, $\sigma_{x x}$ and $\sigma_{x y}$, when plotted versus electron density. However, a direct comparison with macroscopic conductivities is delicate, since the ensemble averaging procedure that we are using may not be appropriate to the thermal averaging taking place in macroscopic experimental samples.

From scaling arguments, one expects that the mean conductances, $\bar{G}(L, \Delta)$, should be only a function of the single scaling parameter $L^{1 / \nu} \Delta$ for large enough $L$ and small enough $\Delta$. Here $\nu$ is the localization length critical exponent, which has a value $\nu \approx 7 / 3$. In Fig. 3 , the the data for $\bar{G}$ in Fig. 2, are replotted versus the scaling variable $L^{1 / \nu} \Delta$, with $\nu=7 / 3$. Although the smaller sizes show marked finite-size scaling corrections, the data collapse is satisfactory at the larger sizes.

It is amusing that with periodic boundary conditions, $\bar{G}$ at the plateau transition $\Delta=0$, is very close to $1 / 2$ for the largest sizes, the same value as the experimentally measured macroscopic $\sigma_{x x}$. But this is probably coincidental, since our averaging procedure is not appropriate for macroscopic samples. With open boundary conditions, the mean conductance is not invariant under $\Delta \rightarrow-\Delta$, due to the breaking of particle-hole symmetry by the boundaries. At the plateau transition, $\bar{G}$ is slightly larger than $1 / 2$, roughly 0.65 for the larger sizes. The increase of $\bar{G}(\Delta=0)$ when changing the boundary conditions from periodic to open can presumably be attributed to an additional contribution coming from edge currents.
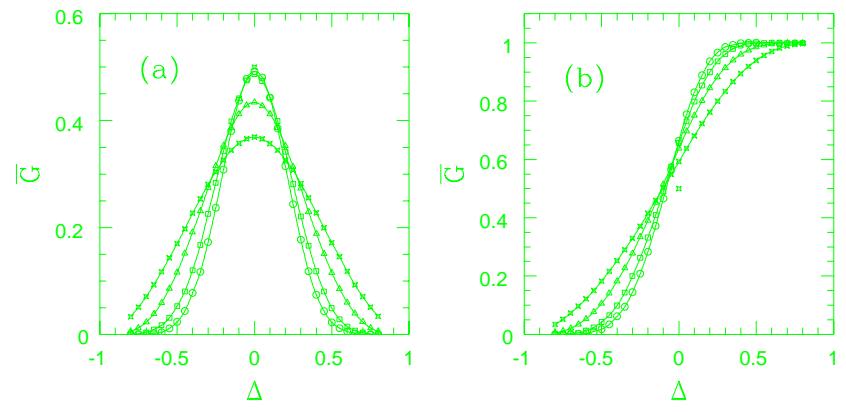

FIG. 2. The mean conductance $\bar{G}$ plotted vs $\Delta$ for square systems of four different sizes $L=4(\times), L=8(\triangle)$, $L=16(\square)$, and $L=24(\bigcirc)$ with (a) periodic and (b) open boundary conditions.
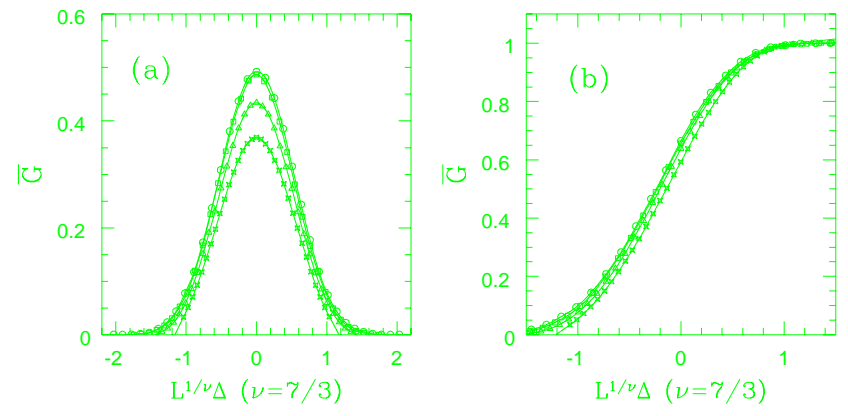

FIG. 3. Scaling collapse of the mean conductance from Fig. 2, plotted vs the parameter $\Delta L^{1 / \nu}$ with $\nu=7 / 3$, for (a) periodic and (b) open boundary conditions.

To characterize the conductance fluctuations first consider the root-mean-square conductance, defined as

$$
\delta G=\sqrt{\bar{G}^{2}-\bar{G}^{2}}
$$

where the overbar denotes an ensemble average over different samples. In Fig. 4, we plot $\delta G$ versus $\Delta$ for four different sample sizes, with periodic boundary conditions in (a) and open in (b). For both boundary conditions, there are large fluctuations near the plateau transition, with $\delta G(\Delta=0) \approx 0.3$. Away from the transition, the fluctuations drop off rapidly with increasing system size, 
as expected. In both cases, the peaks sharpen with increasing $L$, as expected from finite size scaling. The physical origin of the slight double-peaked structure in Fig. $4(\mathrm{a})$ is unclear. In contrast to the mean conductance itself, the root-mean-square conductance is relatively insensitive to finite-size effects at the transition.
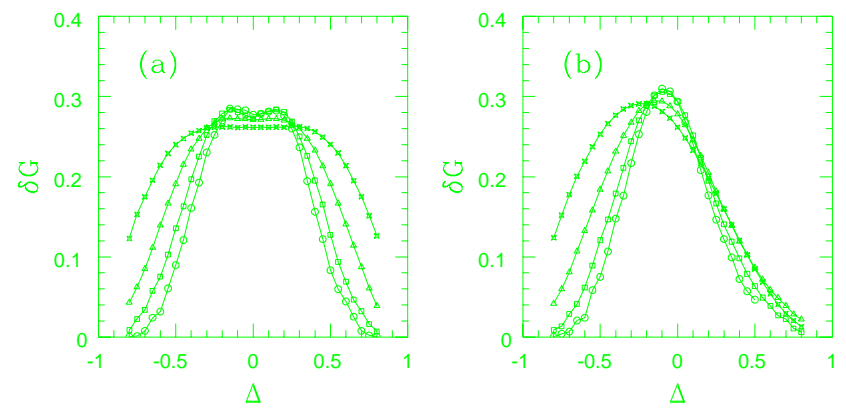

FIG. 4. Root mean square conductance $\delta G$ plotted vs $\Delta$ for a square system of four different sizes: $L=4(\times)$, $L=8(\triangle), L=16(\square)$, and $L=24(\bigcirc)$ with (a) periodic and (b) open boundary conditions.

More informative than the root-mean-square conductance, is the full conductance distribution function, denoted $P(G)$. To obtain this, we simply make a histogram plot of the number of samples with conductance $G$, for a very large ensemble. In Fig. 5, we plot the conductance distribution function at the plateau transition $(\Delta=0)$, for the largest system size $L=24$, with (a) periodic and (b) open boundary conditions. In both cases, the conductance is very broadly distributed, roughly uniform over the interval from zero to one $e^{2} / h$. Above $G=e^{2} / h$, the distribution functions drop off rapidly, although there is a slightly larger "tail" with open boundary conditions, presumably due to edge current contributions.
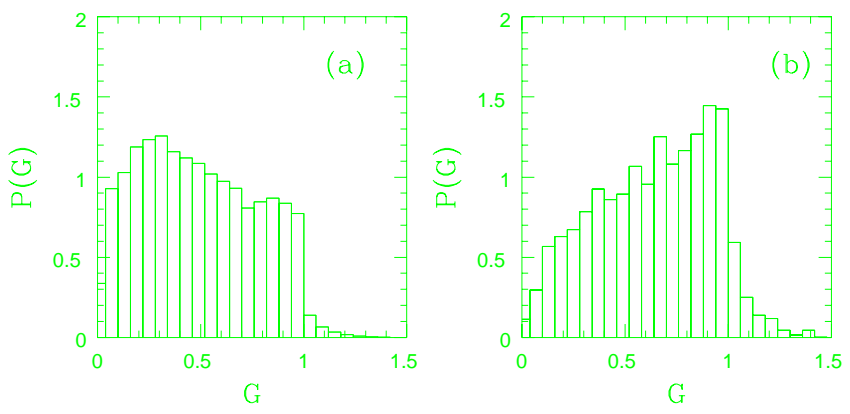

FIG. 5. Conductance distribution function right at the plateau transition, $\Delta=0$, for the system size $L=24$ with (a) periodic and (b) open boundary conditions. The distribution functions are normalized to unity.

\section{DISCUSSION}

The conductance distribution functions obtained numerically compare favorably with those measured in the Cobden and Kogan experiment on a mesoscopic Hall system. In this experiment, the two-terminal conductance of a small sample $\left(0.6 \times 0.6 \mu \mathrm{m}^{2}\right)$ was measured as a function of carrier density, by varying a gate potential $V_{g}$. Large fluctuations in the conductance were seen upon varying $V_{g}$ through the plateau transitions, as shown in Fig. 6(a). In the Hall plateaus themselves, smaller fluctuations were observed. This behavior is consistent with our numerics for the root-mean-square fluctuations, $\delta G$ in Fig. 4, which are largest at the plateau transition. From the data with gate voltages near the plateau transition, Cobden and Kogan obtained a conductance distribution function, shown in Fig. 6(b), which is roughly uniform on the interval from zero to one. Under the "ergodic" assumption that varying the gate potential is equivalent to changing the impurity configuration (i.e., the sample), we can compare their distribution function with ours, which was obtained by taking an ensemble average at the plateau transition (see Fig. 5). The similarity is striking.
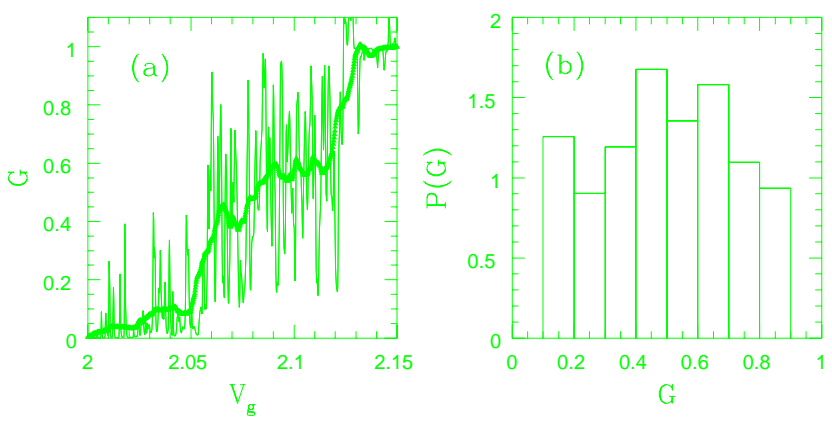

FIG. 6. Cobden and Kogan's experimental data: (a) Two-terminal conductance plotted vs the gate voltage $V_{g}$. The thick solid line is the same data averaged over a $V_{g}$ interval of $16 \mathrm{mV}$. (b) Conductance distribution of data points near the plateau transition, in the interval $2.06 \leq V_{g} \leq 2.12$.

To more closely mimic the experimental procedure, we have computed the conductance for a given sample, as a function of $\Delta$. This is shown in Fig. 7, for a square system of size $L=24$ with both periodic and open boundary conditions. Notice the very large fluctuations in the transition region. The behavior in Fig. $7 \mathrm{~b}$ with open boundary conditions is very similar to the "raw" experimental data of conductance versus gate potential.

In addition to extracting conductance fluctuations for square samples, we have studied systems with various different aspect rations, $L_{x} / L_{y}$. For aspect rations between roughly $1 / 3$ and 3 , the qualitative results are essentially unmodified. For very long sample, however, $L_{x}>>L_{y}$ we start seeing effects of one-dimensional localization. 

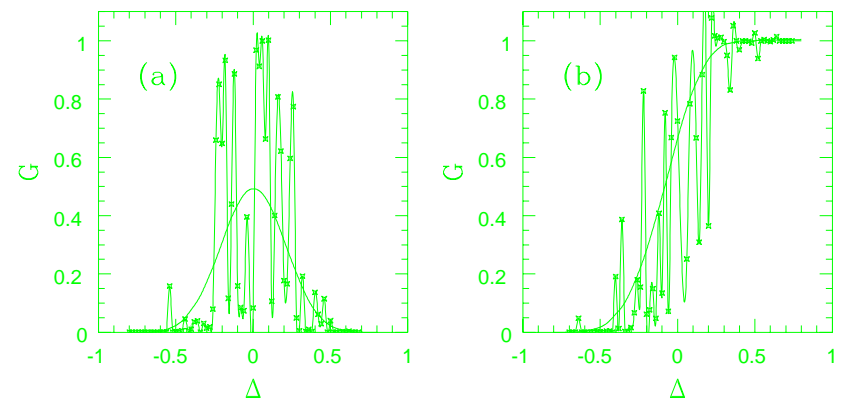

FIG. 7. Conductance plotted vs $\Delta$ of a single sample for the system size $L=24$ with (a) periodic and (b) open boundary conditions. The solid lines are the mean conductance, which was obtained by taking an ensemble average.

In summary, it appears that the quantum Hall plateau transition provides an ideal arena for studying finite-size effects on random phase transitions. The agreement between simple models of non-interacting electrons, and the experimental data is striking. Among the open issues is the role of Coulomb interactions, which have been ignored in the numerics. Will they change the critical behavior, and possibly modify the conductance fluctuations? Moreover, even without interactions, an analytic description of the transition is lacking, for either fluctuations or average properties. One can only hope that the relative experimental accessibility of the quantum Hall plateau transition, will spur further theoretical developments.

\section{ACKNOWLEDGMENTS}

We thank David H. Cobden for generously sharing his experimental data. It is a pleasure to acknowledge Nathan Argaman for fruitful conversations. We are grateful to the National Science Foundation for support under Grants No. PHY94-07194, No. DMR-9400142, and No. DMR-9528578.

${ }^{1}$ Solid State Physics, edited by H. Ehrenreich and D. Turnbull (Academic Press, New York, 1991), Vol. 44 pp. 1-228.

${ }^{2}$ Mesoscopic Phenomena in Solids, edited by B. L. Altshuler, P. A. Lee, and R. A. Webb (North-Holland, New York, 1991).

${ }^{3}$ P. A. Lee, A. D. Stone, and H. Fukuyama, Phys. Rev. B 35, 1039 (1987), and references therein.

${ }^{4}$ The Quantum Hall Effect, 2nd ed., edited by R. E. Prange and S. M. Girvin (Springer-Verlag, New York, 1990).

${ }^{5}$ B. Huckestein, Rev. Mod. Phys. 67, 357 (1995), and references therein.

${ }^{6}$ D. Shahar et al., Phys. Rev. Lett. 74, 4511 (1995).

${ }^{7}$ Y. Huo, R. E. Hetzel, and R. N. Bhatt, Phys. Rev. Lett. 70, 481 (1993).

${ }^{8}$ S. A. Kivelson, D.-H. Lee, and S.-C. Zhang, Phys. Rev. B 46, 2223 (1992).

${ }^{9}$ D. B. Chklovskii and P. A. Lee, Phys. Rev. B 48, 18060 (1993).

${ }^{10}$ D. H. Cobden and E. Kogan, cond-mat/9606114 (unpublished).

${ }^{11}$ After this work was completed we learned of a paper by Wang et al., which also studies conductance fluctuations at Integer Hall Plateau transitions and arrives at similar conclusions. Z. Wang, B. Jovanovic, and D.-H. Lee, condmat/9608111 (unpublished).

12 See also U. Fastenrath, M. Janssen, and W. Pook, Physica A 191, 401 (1992), who studied a Thouless number and found it broadly distributed at criticality.

13 J. T. Chalker and P. D. Coddington, J. Phys. C 21, 2665 (1988).

14 D.-H. Lee, Z. Wang, and S. Kivelson, Phys. Rev. Lett. 70, 4130 (1993).

15 T. Jungwirth and A. H. MacDonald, Phys. Rev. B 53, 7403 (1996).

16 D.-H. Lee and Z. Wang, Phys. Rev. Lett. 76, 4014 (1996).

${ }^{17}$ D. S. Fisher and P. A. Lee, Phys. Rev. B 23, 6851 (1981). 